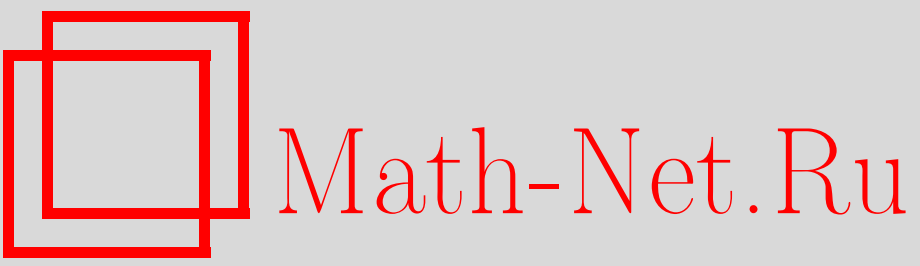

М. А. Чешкова, Обмотки тора и модели проективной плоскости, Итоги науки и техн. Сер. Соврем. мат. и ее прил. Темат. обз., 2020, том 181, 118-120

DOI: https://doi.org/10.36535/0233-6723-2020-181-118-120

Использование Общероссийского математического портала Math-Net.Ru подразумевает, что вы прочитали и согласны с пользовательским соглашением

http://www.mathnet.ru/rus/agreement

Параметры загрузки:

IP: 54.198 .64 .247

26 апреля 2023 г., 13:15:02 


\title{
ОБМОТКИ ТОРА И МОДЕЛИ ПРОЕКТИВНОЙ ПЛОСКОСТИ
}

\author{
(c) 2020 г. M. A. ЧЕШKОBA
}

АннотАция. При помощи периодических вектор-функций построены примеры моделей проективной плоскости.

Ключевые слова: тор, скрещенный колпак, римская поверхность.

\section{WINDINGS OF TORI AND MODELS OF THE PROJECTIVE PLANE}

\section{(c) 2020 M. A. CHESHKOVA}

Abstract. Using periodic vector-valued functions, we construct examples of models of the projective plane.

Keywords and phrases: torus, cross-cap, Roman surface.

AMS Subject Classification: 51A35

1. Введение. Если на поверхности в $E^{3}$ существует замкнутая кривая (дезориентирующий контур), обладающая тем свойством, что при ее обходе локальная ориентация в касательном пространстве меняет знак, то поверхность называется односторонней.

Впервые уравнение односторонней поверхности, открытой Мебиусом, было получено Машке (см. [7]). Если гауссова кривизна листа Мебиуса равна нулю, то он называется плоским. Библиография работ на эту тему дана в [4]. К односторонним поверхностям относятся: скрещенный колпак, римская поверхность, поверхность Боя, бутылка Клейна (см. 3). Римская поверхность, поверхность Боя и скрещенный колпак с крышкой являются моделями проективной плоскости. Односторонние поверхности исследуются автором в [5,6].

2. Построение моделей проективной плоскости. В евклидовом пространстве $E^{3}$ рассмотрим гладкую замкнутую неплоскую кривую $\gamma$ без самопересечения, заданную $4 \pi$-периодической вектор-функцией $\rho=\rho(u)$, которая не является $2 \pi$-периодической и $2 \pi$-антипериодической. Так как $\rho(u)=\rho(u+4 \pi)$, то функция

$$
s(u)=\frac{1}{2}(\rho(u)+\rho(u+2 \pi))
$$

является $2 \pi$-периодической и отличной от нуля, а вектор-функция

$$
l(u)=\frac{1}{2}(\rho(u)-\rho(u+2 \pi))
$$

является $2 \pi$-антипериодической. С помощью этих функций построим модели проективной плоскости.

Теорема 1. Поверхность $\mathbf{P}$, заданная уравнениями

$$
r(u, v)=(1+\cos v) s(u)+\sin v l(u), \quad u \in[-\pi, \pi], v \in[-\pi, \pi],
$$

определяет модель проективной плоскости. 

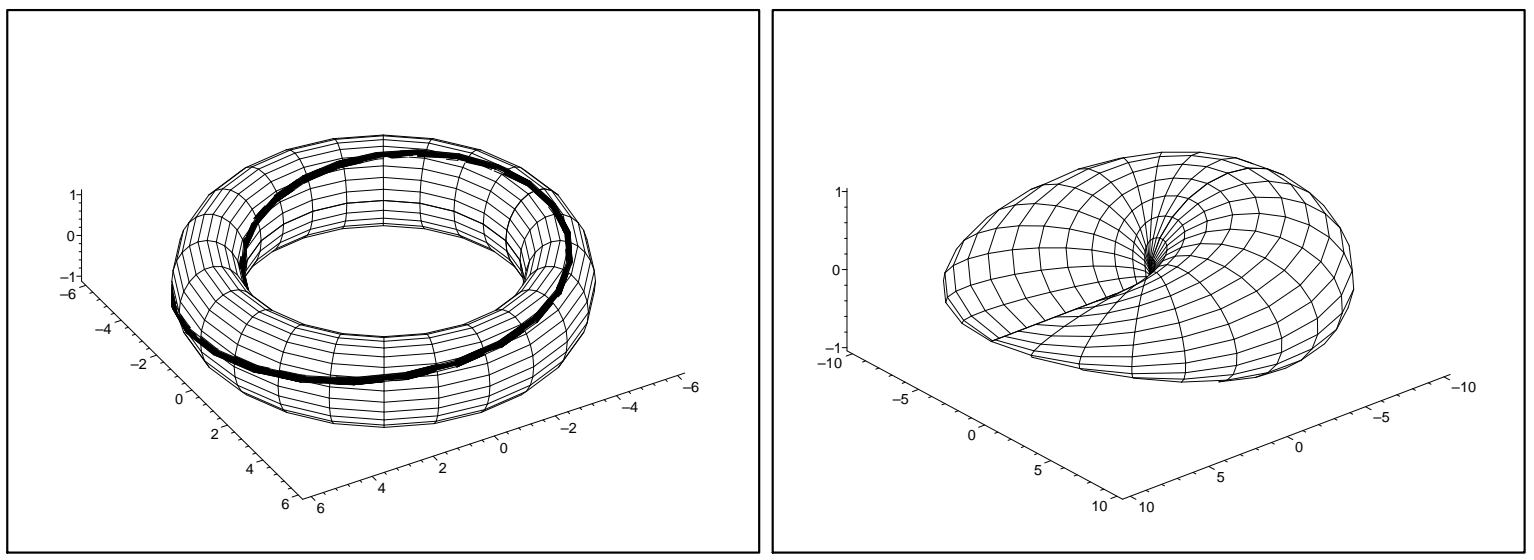

Рис. 1. Обмотка тора первого типа и скрещенный колпак
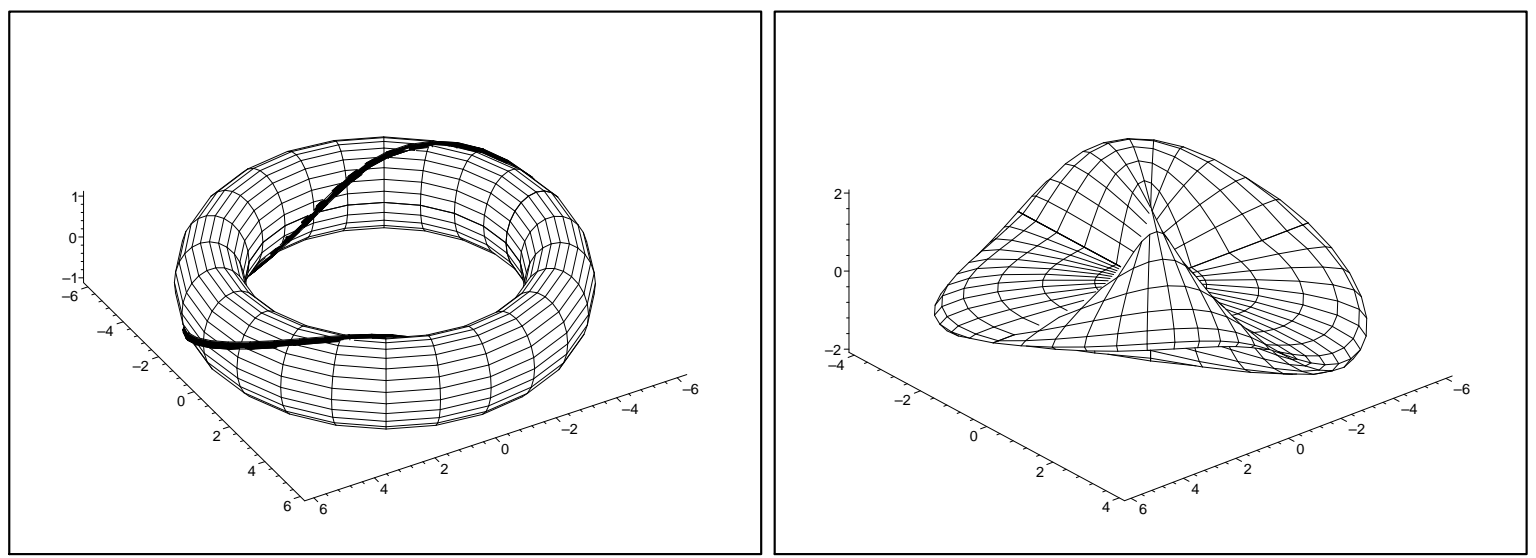

Рис. 2. Обмотка тора второго типа и римская поверхность

Доказательство. Рассмотрим проективную плоскость как фактор-пространство

$$
P^{*}=[-\pi, \pi] \times[-\pi, \pi] /[(-\pi,-v) \sim(\pi, v),(-u,-\pi) \sim(u, \pi)]
$$

(см. [1, с. 75]). Так как

$$
\begin{gathered}
r(\pi, v)=(\cos v+1) s(\pi)+\sin v l(\pi), \\
r(-\pi,-v)=(1+\cos (-v)) s(-\pi)+\sin (-v) l(-\pi), \\
s(-\pi)=s(\pi), \quad l(-\pi)=-l(\pi), \\
r(u, \pi)=(1+\cos \pi) s(u)+\sin \pi l(u)=0, \\
r(-u,-\pi)=(1+\cos (-\pi)) s(-u)+\sin (-\pi) l(-u)=0,
\end{gathered}
$$

то имеем $r(\pi, v)=r(-\pi,-v), r(u, \pi)=r(-u,-\pi)$. Следовательно, поверхность $\mathbf{P}$ есть модель проективной плоскости.

Построим следующие модели проективной плоскости в $E^{3}$ : скрещенный колпак и римскую поверхность. Рассмотрим тор

$$
R(u, v)=(5+\cos v) e(u)+\sin v k, \quad e(u)=(\cos u, \sin u, 0), \quad k=(0,0,1) .
$$

Зададим линию

$$
\left.\rho(u)=R\left(u, \frac{u}{2}\right)=\left(5+\cos \frac{u}{2}\right) e(u)+\sin \frac{u}{2}\right) k .
$$


Назовем ее обмоткой тора первого типа. Имеем

$r(u, v)=(1+\cos v)(5 \cos u, 5 \sin u, 0)+\sin v\left(\cos u \cos \frac{u}{2}, \sin u \cos \frac{u}{2}, \sin \frac{u}{2}\right), \quad u \in[-\pi, \pi], v \in[p i, \pi]$. Поверхность $r=r(u, v)$ называется скрещенным колпаком (см. [3, с. 304]).

Рассмотрим также обмотку тора $\rho(u)=(5+\cos u) e(u / 2)+\sin u k$. Назовем ее обмоткой тора второго типа. Имеем

$$
\rho(u+2 \pi)=-(5+\cos u) e\left(\frac{u}{2}\right)+\sin u k, \quad s(u)=(0,0, \sin u), \quad l(u)=(5+\cos u) e\left(\frac{u}{2}\right) .
$$

Линия $s=s(u)$ у этой поверхности есть отрезок прямой. Такая поверхность называется римской поверхностью Штейнера (см. [2, с. 302], [3, с. 305]). Таким образом, имеет место следующая теорема.

Теорема 2. Если кривая $\rho=\rho(u)$ есть обмотка тора первого типа, то поверхность $\mathbf{P}$ есть скрещенный колпак. Если кривая $\rho=\rho(u)$ есть обмотка тора второго типа, то поверхность $\mathbf{P}$ есть римская поверхность.

Изображение этих поверхностей приведены на рис. 1 и 2).

\section{СПИСОК ЛИТЕРАТУРЫ}

1. Борисович Ю. Г., Близняков Н. М., Израилевич Я. А., Фоменко А. Т. Введение в топологию. - М.: Наука, 1995.

2. Гилъберт Д., Кон-Фоссен С. Наглядная геометрия. - М.: Наука, 1981.

3. Кривошапко С. Н., Иванов В. Н., Халаби С. М. Аналитические поверхности. - М.: Наука, 2006.

4. Сабитов И. Х. Изометрические погружения и вложения плоского листа Мебиуса в евклидовы пространства// Изв. РАН. Сер. мат. - 2007. - № 71 (5). - С. 197-224.

5. Чешкова М. А. Односторонние поверхности// Изв. Алтайск. гос. ун-та. - 2015. - № 1/2 (85). - С. $164-$ 168.

6. Чешкова M. А. Об одной модели бутылки Клейна// Изв. Алтайск. гос. ун-та. - 2016. - № 1 (89). C. $180-184$.

7. Mashke H. Note on the unilateral surface of Moebius// Trans. Am. Math. Sos. - 1900. - 1, № 1 .

Чешкова Мира Артемовна

Алтайский государственный университет, Барнаул

E-mail: cma41@yandex.ru 